\title{
Capturing the Elusive Water Trimer from the Stepwise Growth of Water on the Surface of the Polycyclic Aromatic Hydrocar- bon Acenaphthene
}

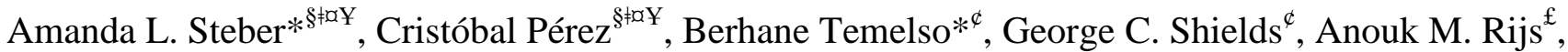

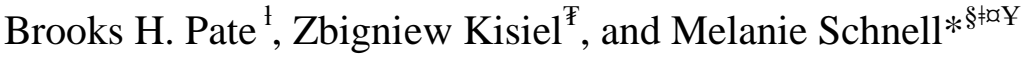 \\ ${ }^{\S}$ Deutsches Elektronen-Synchrotron, Notkestrasse 85, D-22607 Hamburg, Germany \\ ${ }^{\prime}$ Max-Planck-Institut für Struktur und Dynamik der Materie, Luruper Chaussee 149, D-22761 Hamburg, Germany \\ ${ }^{口}$ Institut für Physikalische Chemie, Christian-Albrechts-Universität zu Kiel, Max-Eyth-Strasse 1, D-24118 Kiel, Germany \\ ${ }^{¥}$ The Hamburg Centre for Ultrafast Imaging, Luruper Chaussee 149, D-22761 Hamburg, Germany \\ ${ }^{\phi}$ Provost's Office and Department of Chemistry, Furman University, Greenville, South Carolina 29613, USA \\ ${ }^{\mathfrak{f}}$ Radboud University, Institute for Molecules and Materials, FELIX Laboratory, Toernooiveld 7-c, 6525 ED Nijmegen, The \\ Netherlands \\ ${ }^{1}$ Department of Chemistry, University of Virginia, Charlottesville, Virginia, 22904, USA \\ ${ }^{\mathrm{F}}$ Institute of Physics, Polish Academy of Sciences, PL-02668 Warsaw, Poland
}

KEYWORD: polycyclic aromatic hydrocarbons, rotational spectroscopy, water cluster, ice grain formation

\begin{abstract}
Polycyclic aromatic hydrocarbons (PAHs) are key players in reaction chemistry. While it is postulated that they serve as a basis for ice grains, there has been no direct detection of PAHs in astronomical environments. We aim to investigate the hydration of PAHs in order to set a foundation for the future exploration of potential ice formation pathways. Here, we report results from chirped pulse Fourier transform microwave spectroscopy and quantum chemical calculations for the PAH acenaphthene and acenaphthene complexed with up to four water molecules. The acenaphthene- $\left(\mathrm{H}_{2} \mathrm{O}\right)_{3}$ complex is of particular interest as the elusive cyclic water trimer was observed. It appears in a slightly distorted configuration when compared to the pure water trimer. This is explained by hydrogen bond net cooperativity effects. Binding energies for the complexes are presented. Our results provide insight into the onset of complex aggregation that could be occurring in extraterrestrial environments as part of ice grain formation.
\end{abstract}

Polycyclic aromatic hydrocarbons (PAHs) have long been of interest to scientific communities, from astronomy to catalytic and combustion chemistry. The extent of their presence in interstellar, circumstellar, and star forming regions is unknown. This is mainly due to their high symmetry and lack of dipole moments which prevent the unambiguous identification of PAHs through radio astronomy. However, many believe that their presence is indicated by the diffuse interstellar bands and the unidentified infrared (UIR) bands. To date, most of the bands have not been assigned to a particular $\mathrm{PAH}^{1,2}$, with few exceptions; however, the PAH IR spectroscopic database has been developed in which computationally derived and experimental IR spectra from $2.5-4000 \mu \mathrm{m}$ are stored ${ }^{3}$ to help with the identification of these bands. Analysis of the UIR bands has estimated that PAHs contain up to $20 \%$ of the carbon budget in interstellar space ${ }^{4,5}$.

In these extraterrestrial environments, much about the formation of these stable aromatic systems is unknown. A topdown approach addresses the idea that fullerenes, which have previously been identified ${ }^{6}$ in these environments, larger PAHs, or graphitic grains are destroyed to become PAHs; whereas a bottom-up approach postulates that small hydrocarbons and other small PAHs can react to build up larger PAHs. While both scenarios may occur, one thing that is certain is that the formation of PAHs remains an open question ${ }^{1}$. Regardless of their formation, it is believed that they play an important role in the ongoing chemistry occurring in space ${ }^{1,7}$. They are a source for radical reactions, ion-neutral reactions, and recombination reactions amongst others. Their presence serves to provide a catalyst for organic reactions, as well as the formation of fullerenes ${ }^{8}$ and ice grains ${ }^{4}$.

For these PAHs to contribute to the ice grain population, they would need to interact with gaseous water, after which water aggregates would begin to form on the PAH surfaces. The beginnings of this aggregation can be mimicked in our laboratory on isolated PAH and water molecules in the gas phase and probed using microwave spectroscopy. This technique allows us to determine the experimental structures of the 


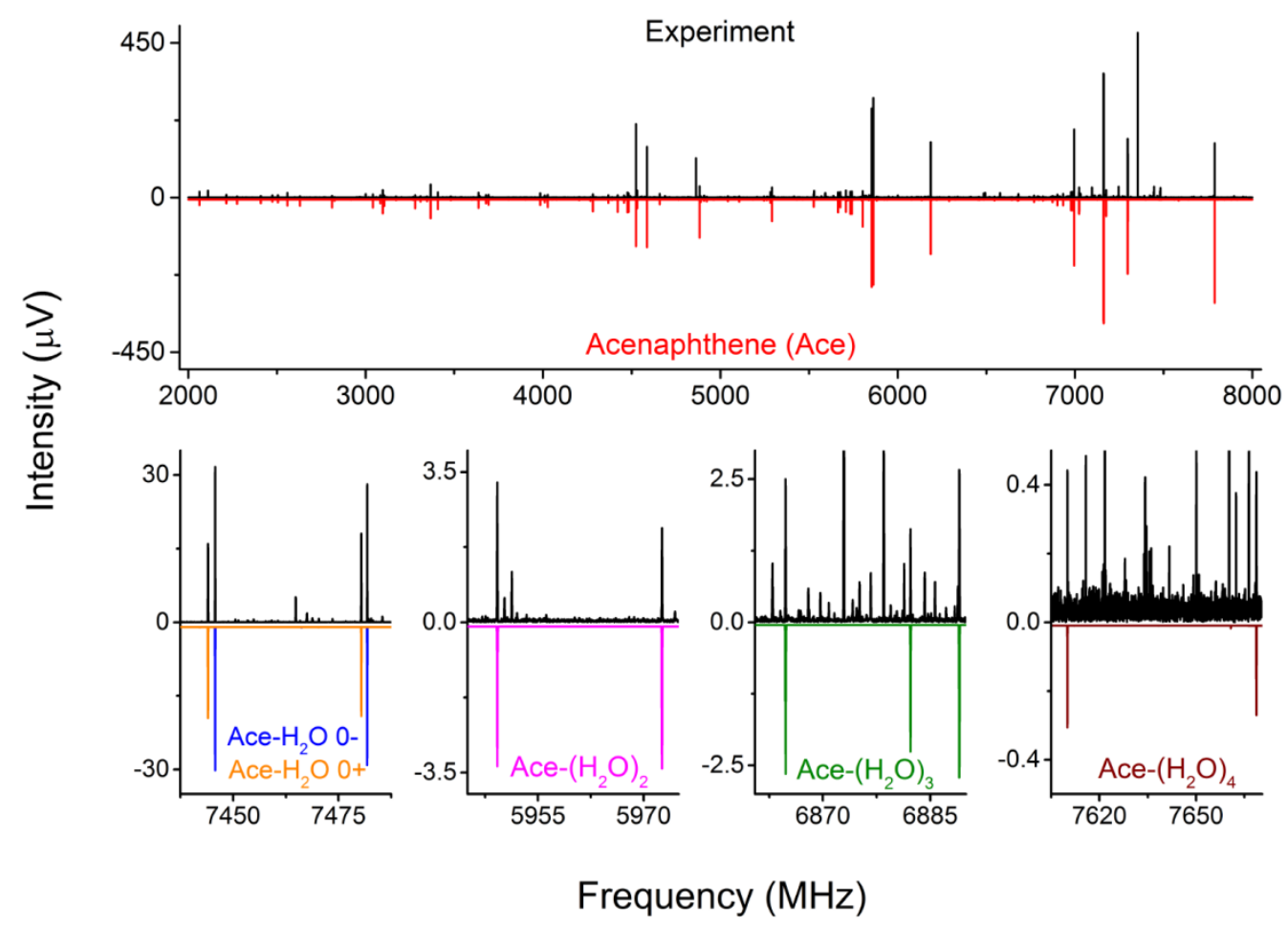

Figure 1: Displayed in the top panel is the 1.4 million average broadband rotational spectrum of acenaphthene with water. The Ace was heated to $100{ }^{\circ} \mathrm{C}$, and 3.5 bar of neon was seeded with water in an external reservoir before picking up the Ace vapor and being expanded into the vacuum chamber. The experimental trace is represented by the black trace, while the fitted parameters for acenaphthene and each complex were used to create the colored simulations. The bottom insets display representative transitions from each of the four complexes, Ace- $\left(\mathrm{H}_{2} \mathrm{O}\right)_{\mathrm{n}=1-4}$. The splitting observed in the one water complex can be seen by the blue and orange traces, which represent the two states arising from the internal motion of the water.

stepwise addition of water molecules to their organic substrate and monitor whether water motifs bound to the PAH surface maintain their structural integrity when compared to pure water clusters $^{9-17}$ and previously studied systems, such as the benzene-water complex ${ }^{18,19}$ and the corannulene-water complex $^{20}$. This gives us insight into the beginnings of how ice would aggregate on a PAH grain surface in the gaseous environments of astronomical media.

We report the structural results of the PAH acenaphthene (Ace) and Ace-water complexes by the highly sensitive and high-resolution method of chirped pulse Fourier transform microwave (CP-FTMW) spectroscopy ${ }^{21,22}$ in the $2-8 \mathrm{GHz}$ region. In this broadband technique where fast acquisition times and low sample consumption requirements are common, complexes are formed and then rotationally cooled to $1-2 \mathrm{~K}$ in a supersonic expansion. The molecular fingerprint characteristic of rotational spectroscopy that allows us to observe all present polar complexes simultaneously arises from the fact that even small changes in the moments of inertia upon isotopic substitution or conformational variations significantly change the spectrum.

Taking advantage of this, structural determination from experimental data has become common place in CP-FTMW studies through use of the Kraitchman equations ${ }^{23}$ and/or leastsquares fitting procedures ${ }^{24,25}$.The Kraitchman equations exploit the shifts in the moments of inertia to determine the coordinates of the isotopically substituted atom in the principal coordinate axis system, allowing one to build up the substitu- tion $\left(r_{\mathrm{s}}\right)$ structure atom by atom. In the least-squares fitting routines, the effective ground-state $\left(r_{0}\right)$ structure can be determined from the experimental data with help from ab initio calculations. In the cases where vibrational-rotational effects occur, additional parameters are added to the fit and the experimental equilibrium structure, $r_{\mathrm{m}}{ }^{(1)}$, is obtained. All three methods are complimentary and have been employed for many complex, pure water cluster studies ${ }^{12-14}$.

The rotational spectrum of Ace has previously been assigned by microwave spectroscopy ${ }^{26}$; however, they reported no experimental structural data. This information is crucial for understanding structural differences incurred upon complexation with water. We have extended the previous spectroscopic investigation and experimentally determined the Ace monomer structure. The broadband spectrum is shown in Fig. 1 top panel.

The strongest rotational transitions of Ace had a signal to noise ratio of $\sim 850: 1$ so we were able to measure six of the seven ${ }^{13} \mathrm{C}$ mono substituted spectra in natural abundance. The seventh ${ }^{13} \mathrm{C}$ resides close to the center of mass, and its spectrum is suspected to be beneath the spectrum of the parent species. By fitting the rotational spectra of the ${ }^{13} \mathrm{Cs}$, the $r_{\mathrm{m}}{ }^{(1)}$, $r_{0}$, and $r_{\mathrm{s}}$ experimental structures were determined. The rotational constants of the monomer suffer from vibration-rotation contributions, likely due to the low energy vibrationally excited state at $\sim 100 \mathrm{~cm}^{-1}$ above the ground state, and thus the $r_{\mathrm{m}}{ }^{(1)}$ structure best represents the experimental structure. The $r_{\mathrm{m}}{ }^{(1)}$ structure overlaid with the $r_{\mathrm{s}}$ structure and their bond distances 
are shown in Fig. 2. Bond distances from both experimental structures agree to within $0.01 \AA$, ranging from $1.38-1.57 \AA$ with the three non-aromatic bonds being the longest. All experimental data pertaining to Ace and the determination of its structure can be found in supplementary materials section 1.

Upon complexation of Ace with water, the clusters Ace$\left(\mathrm{H}_{2} \mathrm{O}\right)_{n}$ with $\mathrm{n}=1-4$ were identified in the broadband spectrum. Examples of each spectrum are shown in Fig. 1 - bottom panels. ${ }^{13} \mathrm{C}$ spectra for the one-water complex and mono ${ }^{18} \mathrm{O}$ substitution spectra for the Ace- $\left(\mathrm{H}_{2} \mathrm{O}\right)_{\mathrm{n}=1-3}$ complexes can be seen in Supplementary Fig. 5 and 6. Splitting attributed to internal dynamics of the water, as explained thoroughly by Evangelisti and Caminati ${ }^{27}$, was seen for the one-water complex. This is a common feature seen in pure water complexes ${ }^{15}$, and it was most recently investigated by Richardson et al. ${ }^{28}$. Both states $\left(0^{+}\right.$and $\left.0^{-}\right)$arising from the internal motion of the water in the Ace- $\mathrm{H}_{2} \mathrm{O}$ were investigated independently. This splitting was not observed for the higher order Ace-water complexes, indicating that the mobility of the water is notably reduced by their strong hydrogen bond networks.

The experimental structures for the first three complexes and the ab initio structure for the four-water complex are shown in Fig. 3a-d. After complexation the water moiety maintains a similar structure to its analogous pure water cluster. The water molecules complex in a plane nearly parallel (at an angle of $0.7(4)^{\circ}$ for the Ace- $\left(\mathrm{H}_{2} \mathrm{O}\right)_{2}$ complex and $1.0(4)^{\circ}$ for the Ace- $\left(\mathrm{H}_{2} \mathrm{O}\right)_{3}$ complex $)$ to the planar surface of Ace as is evident by the similar perpendicular distances between the water $\mathrm{O}$ atoms and the Ace plane. It was also revealed that the measured rotational constants for the complexes are influenced by vibration-rotation contributions, as was found for the Ace monomer. Thus the $r_{\mathrm{m}}{ }^{(1)}$ structure best represents the experimental structure of the complexes as it accounts for these contributions. However, the three-water complex has insufficient moments of inertia for an $r_{\mathrm{m}}{ }^{(1)}$ least-squares fitting so the $r_{0}$ structure is presented. Supplementary materials section 2 contains all information pertaining to these complexes and their structural determination.

Previously reported structures of small organic moleculewater clusters show a variety of water-water and watersubstrate interactions. One of the best comparisons for the one-water complex is the example of benzene-water. In the complex, the water binds to benzene $3.396 \AA$ away from the benzene plane ${ }^{18,19}$. Benzene acts as a hydrogen bond acceptor, while the water sits directly over its $\pi$ cloud. The water's $C_{2}$ axis is tilted by $35^{\circ}$ with respect to benzene so that the protons of the water are not pointing directly at the aromatic surface ${ }^{18,19}$. This is contrary to the Ace- $\mathrm{H}_{2} \mathrm{O}$ complex. Like the benzene-water complex, the water molecule sits above the $\pi$ cloud of Ace; however, the distance between the O-atom and the plane of Ace is only $3.279 \AA$ ( $0.09 \AA$ closer $)$. Both protons are calculated to be directly interacting with the aromatic $\pi$ cloud of Ace with the C2 axis of the water tilted by only $2.5^{\circ}$ to Ace. This is much like another PAH-water cluster, corannulene-water, where the water molecule sits $3.32 \AA$ above the plane of the central carbon pentagon of corannulene, and the hydrogens point directly towards corannulene ${ }^{20}$ (Fig. 3e). The increased binding energies of the water molecule to the two PAHs (see below for further information) and the higher directionality of the water protons help to account for the shorter

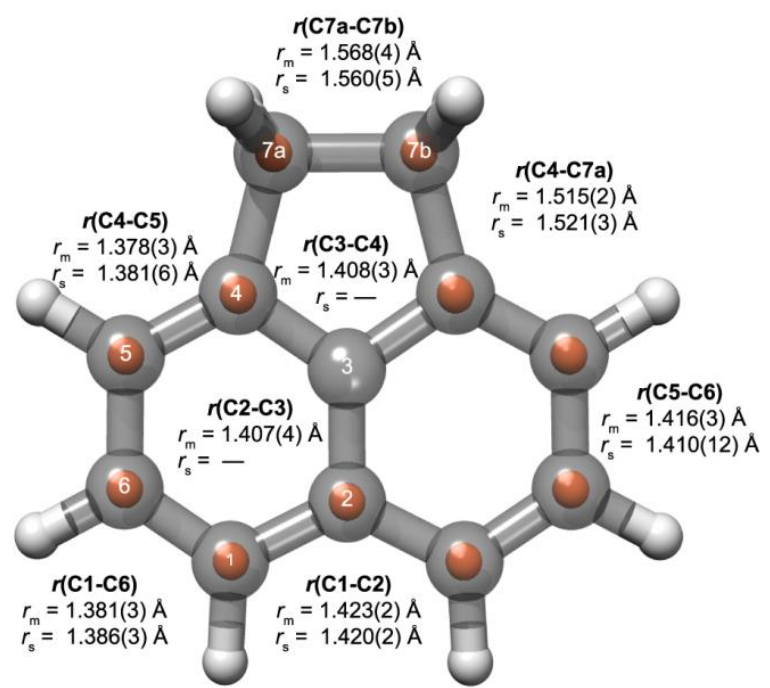

Figure 2: The experimental structures of the Ace monomer: the ball and stick structure is the least-squares fitted $r_{\mathrm{m}}{ }^{(1)}$ structure while the orange spheres represent the experimentally determined substitution, $r_{\mathrm{s}}$, atom positions. Determined bond distances for both experimental structures are shown. The C3 atom lies very close to the center of mass so that the ${ }^{13} \mathrm{C} 3$ isotopic spectrum could not be assigned since it is estimated to be directly overlapped by that of the much stronger parent species.

O-PAH plane distance. For the two-water complex, anchoring C-H...O interactions form between the water oxygen sitting over the non-aromatic portion of Ace and Ace's out-of-plane (OOP) hydrogens. Both water molecules are found to be in the ac inertial plane of the complex, approximately $3.17 \AA$ above the Ace plane. $\mathrm{Ab}$ initio calculations indicate that in the lowest energy form of this complex, the water dimer is slightly skewed from the $a c$ inertial plane, but the observed position corresponds to the vibrational average of two equivalent minima. More information can be found in the supplementary materials section 2.2 .

The pure water trimer is the smallest cyclic water cluster, and it has been measured by terahertz/far-IR vibrational rotational tunneling spectroscopy ${ }^{16,17}$. However, the rapidly interchanging protons cause a lack of a permanent dipole moment in the trimer, making this one of the pure water complexes that cannot be measured by microwave spectroscopy. Only a single microwave spectroscopic observation of the cyclic water trimer, in which it formed a van der Waals complex with $\mathrm{Ar}^{29}$, has been published. The present study reports the first high resolution observation of the cyclic water trimer bound to an organic molecule. In the observed complex, two of the waters reside over the aromatic portion and interact with the $\pi$ cloud of the PAH while the third water is engaged in C-H...O anchoring interactions with the OOP hydrogens of Ace, see Fig. $3 \mathrm{c}$. The best fit experimental structure required the dangling protons (those that are not involved in a hydrogen bond) to be oriented in a down-up-up (DUU) orientation relative to Ace. However, ab initio calculations found the DDU water trimer isomer to be lowest in energy by $\sim 12 \mathrm{~kJ} \mathrm{~mol}^{-1}$ (Supplementary Fig. 16). The discrepancy between the calculated and experimental structure can be attributed to the internal dynamics of the water subunits, which contributes significantly to the experimentally observed vibrationally averaged structure. 


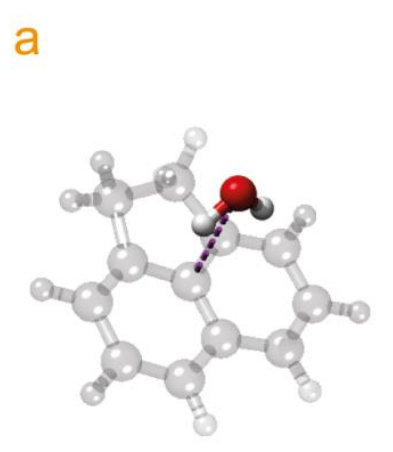

$\perp(\mathrm{O} . \mathrm{Ace})=3.28(1) \AA$

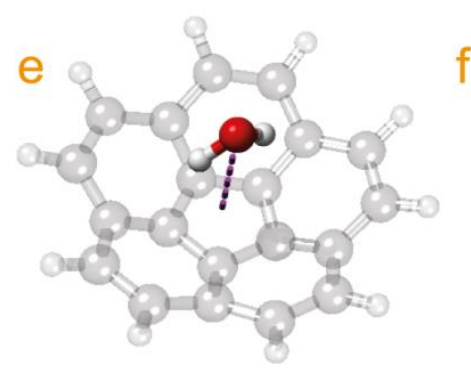

$\perp(\mathrm{O} . \mathrm{Cor})=3.32 \AA$

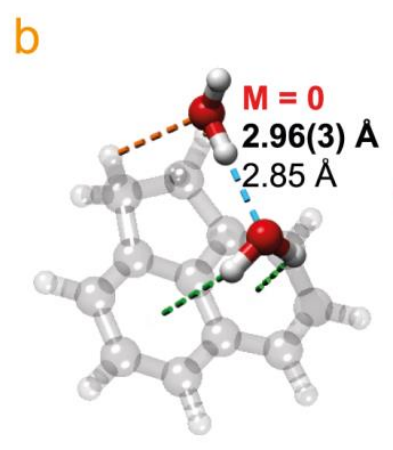

$\perp(01$. Ace $)=3.18(2) \AA$

$\perp($ O2.Ace $)=3.15(2) \AA$

f

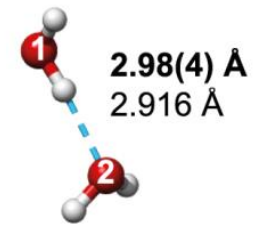

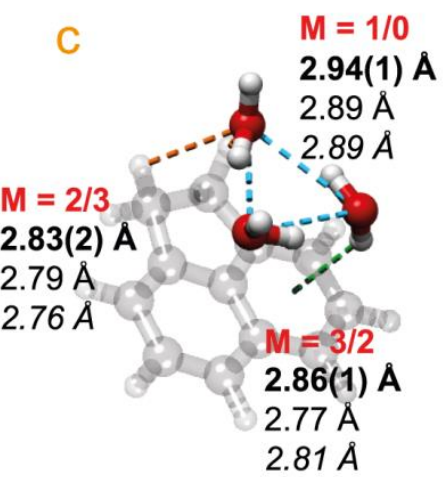

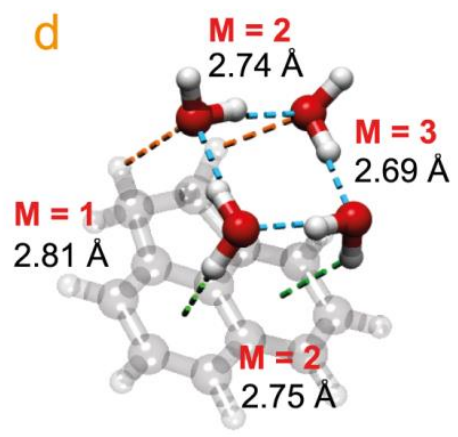

$\perp($ O1.Ace $)=3.25(2) \AA$

$\perp($ O2.Ace $)=3.29(3) \AA$

$\perp($ O3.Ace $)=3.24(3) \AA$

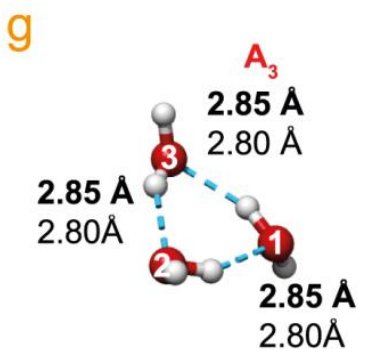

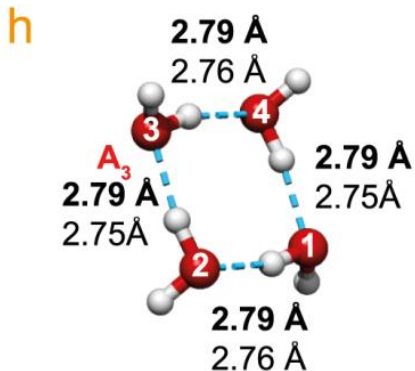

Figure 3: Structures and the manifestation of net cooperativity effects on the Ace- $\left(\mathrm{H}_{2} \mathrm{O}\right)_{\mathrm{n}=1-4}$ complexes. The $r_{\mathrm{m}}{ }^{(1)}$ and $\mathrm{r}_{0}$ experimental structures are illustrated for Ace- $\left(\mathrm{H}_{2} \mathrm{O}\right)_{\mathrm{n}=1-2}$ [a and b] and Ace- $\left(\mathrm{H}_{2} \mathrm{O}\right)_{3}$ [c] respectively, along with the O-O distances determined experimentally (bold) and from ab initio calculations (non-bolded). In the Ace- $\left(\mathrm{H}_{2} \mathrm{O}\right)_{3}$ complex, the italicized values indicate the DDU isomer and the non-italicized values are the ab initio values for the DUU structure. For the one water complex, the average rotational constants of the two states, $0^{+}$and $0^{-}$, were used in order to determine the experimental oxygen atom position. The perpendicular distances of the water oxygens to the Ace plane are displayed for the experimentally determined structures, and the one water complex is compared to the structure observed for the corannulene-water complex ${ }^{20}[\mathrm{e}]$. The ab initio structure for the Ace- $\left(\mathrm{H}_{2} \mathrm{O}\right)_{4}$ complex is shown [d]. All calculations were computed at the MP2/aug-cc-pVDZ level of theory. The value of the $M$ cooperativity parameter listed for each O-O distance in the Ace- $\left(\mathrm{H}_{2} \mathrm{O}\right)_{\mathrm{n}=2-4}$ clusters is seen to be in good inverse correlation with $\mathrm{O}-\mathrm{O}$ distances and well describes the O-O shortening or elongation relative to the equal distances in the pure water clusters $[\mathrm{g}-\mathrm{h}]$, where all $\mathrm{O}-\mathrm{O}$ bonds are characterized by $M=2$. For Ace- $\left(\mathrm{H}_{2} \mathrm{O}\right)_{3}$ the $M$ value for the DUU/DDU isomers

are

given.

The remaining observed complex involves a cyclic water tetramer interacting with the Ace substrate. For $\left(\mathrm{H}_{2} \mathrm{O}\right)_{4}$, the UDUD configuration has been calculated to be the lowest energy isomer, and it is the one that has been estabilished ${ }^{15}$. However, when the water tetramer complexes with Ace, ab initio calculations show that the lowest energy conformation becomes DDUU. Similarly, a distorted DDUU water tetramer complexed with the organic molecule $\beta$-propiolactone (BPL) ${ }^{30}$ has been observed by microwave spectroscopy. For BPL the polar groups are the binding sites of choice, which force the water tetramer to become significantly distorted. In the Ace complex, the interactions do not change much from those of the three-water complex, except that the fourth water molecule utilizes the second OOP proton on Ace to form an anchoring C-H...O interaction. These two C-H...O interactions force the DDUU configuration and anchor the water tetramer to the substrate. It appears that water's affinity for itself compared to the weak Ace-water interactions cause the water motifs to hardly vary from those of the pure water clusters.

To monitor the effects of the Ace substrate on the pure water cluster, Table 1 displays the O-O distances of the experimental structures (Fig. 3b-d) compared to those of the pure water clusters (Fig. 3f-h). This gives us an indication of induced distortions. For the four-water complex, this was done using ab initio values as experimental structural information could not be obtained for this complex. We see that the pure water dimer has an O-O experimental distance of 2.98(4) $\AA^{11}$, whereas the $\mathrm{O}-\mathrm{O}$ distance in Ace- $\left(\mathrm{H}_{2} \mathrm{O}\right)_{2}$ decreases to 2.96(3) $\AA$. This is mirrored in the ab initio calculations. In the case of the completely symmetric pure water trimer, the experimental $\mathrm{O}-\mathrm{O}$ distances are $2.85^{15}$. However, for the Ace- $\left(\mathrm{H}_{2} \mathrm{O}\right)_{3}$ complex we see that the symmetry is broken. If we consider O1$\mathrm{O} 2$ to be the average experimental O-O distance at 2.86(1) $\AA$, O2-O3 becomes shorter (2.83(2) $\AA$ ), while O1-O3 is longer (2.94(1) $\AA$ ). The C-H...O and O-H... $\pi$ interactions collectively anchor the trimer to the Ace surface. This shows how flexible but persistent the water trimer can be.

In the pure water UDUD tetramer, ab initio calculations predict that the parallel bonds have the same bond length: 2.76 and $2.75 \AA$. However, in BPL- $\left(\mathrm{H}_{2} \mathrm{O}\right)_{4}$ one set of perpendicular bond lengths are the same at 2.75(2) $\AA$ while the other two are 2.68(7) $\AA$ and 2.94(8) $\AA$. This trend is not calculated for the Ace complex, especially since there are no polar groups, as the parallel bonds complement each other. As in the three-water 
Table 1: The oxygen-oxygen distances $(\AA)$ for the pure water complexes and those of the Ace-water complexes for two to four water molecules. The ab initio values were obtained from calculations at the MP2/aug-cc-pVDZ level of theory, and the experimentally determined $\mathrm{O}-\mathrm{O}$ distances are shown for a variety of methods. The atom labels $(1,2$, and 3$)$ can be found in Fig. 3.

\begin{tabular}{llllll} 
& Water Dimer & \multicolumn{3}{c}{ Ace- $\left(\mathbf{H}_{\mathbf{2}} \mathbf{O}\right)_{\mathbf{2}}$ Complex } \\
\hline & ab initio & $\exp ^{11}$ & ab initio & $\boldsymbol{r}_{\mathbf{m}}{ }^{(1)}$ structure & $\boldsymbol{r}_{\mathbf{s}}$ structure \\
\hline $\mathbf{O 1 - O 2}$ & 2.916 & $2.98(4)$ & 2.847 & $2.964(26)$ & $3.003(5)$ \\
\hline
\end{tabular}

Water Trimer

Ace- $\left(\mathrm{H}_{2} \mathrm{O}\right)_{3}$ Complex

\begin{tabular}{llllll}
\hline & ab initio & exp $^{15}$ & ab initio $^{a}$ & $\boldsymbol{r}_{\mathbf{0}}$ structure & $\boldsymbol{r}_{\mathbf{s}}$ structure \\
\hline $\mathbf{O 1 - O 2}$ & 2.802 & 2.85 & $2.766 / 2.812$ & $2.856(10)$ & $2.851(11)$ \\
$\mathbf{O 2 - O 3}$ & 2.802 & 2.85 & $2.790 / 2.758$ & $2.825(14)$ & $2.808(12)$ \\
$\mathbf{O 1 - O 3}$ & 2.804 & 2.85 & $2.891 / 2.891$ & $2.944(10)$ & $2.930(12)$ \\
\hline
\end{tabular}

Water Tetramer

\begin{tabular}{llllll}
\hline & ab initio $^{b}$ & $\mathbf{e x p}^{15 c}$ & ab initio & $\boldsymbol{r}_{\mathbf{0}}$ structure & $\boldsymbol{r}_{\mathrm{s}}$ structure \\
\hline $\mathbf{O 1 - O 2}$ & 2.756 & 2.79 & 2.754 & --- & -- \\
$\mathbf{O 2 - O 3}$ & 2.749 & 2.79 & 2.808 & --- & --- \\
$\mathbf{O 3 - O 4}$ & 2.756 & 2.79 & 2.744 & --- & --- \\
$\mathbf{O 1 - O 3}$ & 2.749 & 2.79 & 2.692 & --- & --- \\
\hline
\end{tabular}

${ }^{a} \mathrm{Ab}$ initio values for the DUU isomer and the DDU isomer.

${ }^{b}$ The DDUU isomer O-O distances are shown in this column, even though it is not the lowest energy isomer for the pure water tetramer. A direct comparison can then be made between the pure water tetramer and the Ace- $\left(\mathrm{H}_{2} \mathrm{O}\right)_{4}$ complex.

${ }^{c}$ The experimental O-O distances reported are for the observed water tetramer isomer UDUD $\left(\mathrm{S}_{4}\right)$. This isomer of the tetramer has equal $\mathrm{O}-\mathrm{O}$ distances.

complex, the tetramer is anchored to the Ace substrate via the C-H...O and O-H... $\pi$ interactions. The O-O distance pairs for the Ace complex are $2.74 \AA / 2.75 \AA$ and $2.69 \AA / 2.81 \AA$. These discrepancies seen in the O-O distances for all three complexes compared to their pure water counterparts can be explained by net cooperativity effects ${ }^{31,32}$.

Net cooperativity effects are governed by the local environment of a water molecule. This environment changes as the potential for the water molecule to form hydrogen bonds (HBs) increases or decreases. New HBs, in which the water can be an HB donor, acceptor, or both, cause other hydrogen bonds that it is involved in to strengthen or weaken. For the Ace-water clusters, the C-H...O interactions between the OOP hydrogens of Ace and the water clusters, as well as the $\mathrm{O}$ H... $\pi$ interactions between the $\pi$ cloud of Ace and the free hydrogens of the water cluster serve to anchor the water clusters in significant ways. The strength of the C-H...O interactions with the OOP hydrogens is about $6-8 \mathrm{~kJ} \mathrm{~mol}^{-1}$ in total or about 3-4 $\mathrm{kJ} \mathrm{mol}^{-1}$ per C-H...O. Nevertheless, they are not strong enough to affect the strength and geometry of the hydrogen bonds in the water clusters significantly. However, the $\mathrm{O}-\mathrm{H} \ldots \pi$ interactions are much stronger, as indicated by the fact that benzene- $\mathrm{H}_{2} \mathrm{O}$ 's binding energy is $12.1 \mathrm{~kJ} \mathrm{~mol}^{-1}$ (Table 2). In Ace- $\mathrm{H}_{2} \mathrm{O}$, where the different binding contributions are roughly of the same ratio as in benzene- $\mathrm{H}_{2} \mathrm{O}$, the binding energy is $17.9 \mathrm{~kJ} \mathrm{~mol}^{-1}$. While these $\mathrm{O}-\mathrm{H} . . . \pi$ interactions are weaker than a direct hydrogen bond at $19.1 \mathrm{~kJ} \mathrm{~mol}^{-1}$ for $\left(\mathrm{H}_{2} \mathrm{O}\right)_{2}$, they are effectively serving as hydrogen bond acceptors. One way to gauge the effect of these interactions is through the O-O distance differences observed between the Ace complexes and the pure water moieties.

In order to quantify the cooperative effects, Ohno et al. ${ }^{31}$ defined a parameter $M$ which takes into account potential twobody and three-body HBs of the donor (D) -acceptor (A) pairs. The values of $M$ can range from -2 to 4 , with larger numbers indicating a stronger $\mathrm{HB}$ and a shorter donoracceptor distance $(r[\mathrm{D} . . \mathrm{A}])^{32}$. Figure 3 shows how these cooperativity effects manifested in the Ace- $\left(\mathrm{H}_{2} \mathrm{O}\right)_{2-4}$ clusters (Fig. $3 \mathrm{~b}-\mathrm{d}$ ) compared to $\left(\mathrm{H}_{2} \mathrm{O}\right)_{2-4}$ (Fig. 3f-h) by the O-O distances. In the case of Ace- $\left(\mathrm{H}_{2} \mathrm{O}\right)_{3}$, the two effective $\pi$ clouds of Ace serve as hydrogen bond acceptors. This changes the nearest water molecules (W1 and W2) from waters with only two HB interactions to waters with three $\mathrm{HB}$ interactions. While all HBs in the pure water trimer have a net cooperativity value $M$ $=2$, the $\mathrm{HB}$ between $\mathrm{W} 1$ and $\mathrm{W} 3$ changes to $M=0 / 1$ for the DDU/UUD trimer configuration and the HB between W3 and W2 changes to $M=3 / 2$ due to the influence of Ace. The result is that the W3-W2 HB shortens while the W1-W3 HB elongates. This explains the distortion of the water trimer and the deviation of the O-O distances compared to the complex's pure water counterpart. Similar distortions in O-O distances have been observed in benzene- $\left(\mathrm{H}_{2} \mathrm{O}\right)_{n}, \mathrm{n}=2-7^{33,34}$. Other 

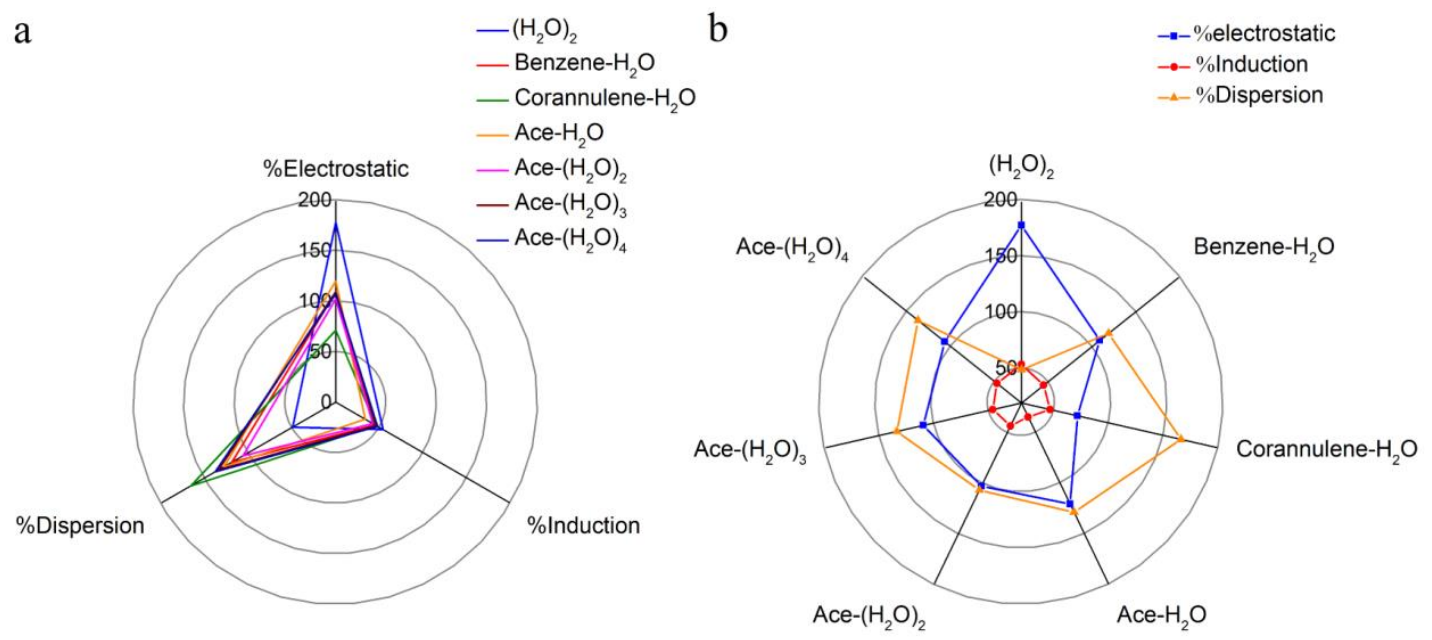

Figure 4. Radar charts showing the breakdown of the attractive components of the two-body SAPT interaction energies reported in Table 2 as a percentage of the total of interaction energy $\left(\Delta \mathrm{E}_{\text {component }} / \Delta \mathrm{E}_{\text {total }}\right)$. The total SAPT2+3/aug-cc-pVDZ interaction energy is the sum of the attractive electrostatic, induction, and dispersion components, and the repulsive exchange contribution which is not shown here. The figure showcases this breakdown by interaction energy (Panel a) and system (Panel b). Both panels show that the percent contribution of the electrostatic component is largest for the hydrogen bonded $\left(\mathrm{H}_{2} \mathrm{O}\right)_{2}$ dimer while dispersion is far more dominant for corannulene- $\mathrm{H}_{2} \mathrm{O}$. Benzene- $\mathrm{H}_{2} \mathrm{O}$ and acenaphthene- $\left(\mathrm{H}_{2} \mathrm{O}\right)_{n}$ exhibit similar behavior with dispersion playing the biggest role closely followed by electrostatics, and with induction playing the smallest r

aspects of net cooperativity in Ace- $\left(\mathrm{H}_{2} \mathrm{O}\right)_{n}$ and $\left(\mathrm{H}_{2} \mathrm{O}\right)_{n}$ clusters are included in the supplementary material section 2.3 .

One of the main questions about weakly bound clusters is how likely would it be for these complexes to persist in an interstellar environment. Dissociation energies were determined using SAPT2 ${ }^{35}$ calculations, described in the methods section in the supplemental materials, and they are presented in Table 2. The SAPT2 method allows us to investigate the different binding contributions in each of the complexes. Since the water complex forms strong HBs within the water network while the overall complex is formed by a weaker dispersive interaction plus weak hydrogen bonding, the complexes can be viewed as two-body systems for these calculations. This implies that it would be more feasible for the water complex to detach from the Ace substrate than for a single water molecule to be removed from the complex. The results are compared to those of the water dimer, the benzene-water complex ${ }^{18,19}$, and the corannulene-water complex ${ }^{20}$, and they are shown pictorially in Fig. 4. In the water dimer system, the electrostatic contribution dominates with $179 \%$ of the total binding contribution, while dispersive interactions dominate in corannulenewater at $167 \%$ of the total binding contribution. For the benzene-water system, the total binding contribution consists of equal parts electrostatic and dispersive interactions.

The Ace-water complex is similar to the benzene-water complex in that both dispersion interactions and hydrogen bonding play a crucial role. The total dissociation energy of the Ace-water complex is higher than that of the benzenewater complex $\left(-17.9 \mathrm{~kJ} \mathrm{~mol}^{-1}\right.$ compared to $\left.-12.1 \mathrm{~kJ} \mathrm{~mol}^{-1}\right)$. This is likely due to the higher electron density of Ace and the fact that both protons of water interact with the $\pi$ cloud in the Ace-water complex more efficiently than in the benzenewater ${ }^{19}$ complex as explained earlier. As expected, the dissociation energy increases as the complexes go up in size, up to -
$43.5 \mathrm{~kJ} \mathrm{~mol}^{-1}$ for the four-water complex. The anchoring of the water complexes to the Ace OOP hydrogens and the weak HBs from the other waters continue to increase the electrostatic and inductive contributions. In Ace- $\mathrm{H}_{2} \mathrm{O}$ the sum of the electrostatic and inductive are comparable to the dispersion contributions. This remains true as we add more waters to the complex. Nevertheless, dispersion interactions are more prominent in the binding of Ace- $\left(\mathrm{H}_{2} \mathrm{O}\right)_{\mathrm{n}=1-4}$ than in smaller prototypical systems like the water dimer. Thus it is easier to dissociate a planar PAH-water complex than it is to dissociate small strongly bound systems like $\left(\mathrm{H}_{2} \mathrm{O}\right)_{2}$. However, as the water moiety increases in size, the complex becomes harder to break apart, which is important for ice grain growth in astronomical environments.

Table 2: Dissociation energies $\left(\mathrm{kJ} \mathrm{mol}^{-1}\right)$ calculated at the SAPT2+3/aug-cc-pVDZ level of theory. These values were calculated using a two-body binding motif, in which the water cluster was considered as a single unit.

\begin{tabular}{llllll} 
& $\boldsymbol{\Delta} \mathbf{E}_{\text {elst }}$ & \multicolumn{1}{c}{$\boldsymbol{\Delta \mathbf { E } _ { \text { ind } }}$} & $\boldsymbol{\Delta} \mathbf{E}_{\text {disp }}$ & $\boldsymbol{\Delta} \mathbf{E}_{\text {exch }}$ & $\boldsymbol{\Delta} \mathbf{E}_{\text {tot }}$ \\
\hline$\left(\mathrm{H}_{2} \mathrm{O}\right)_{2}$ & -34.19 & -10.38 & -9.49 & 35.00 & -19.06 \\
Benzene- $\mathrm{H}_{2} \mathrm{O}$ & -13.31 & -5.51 & -14.51 & 21.25 & -12.09 \\
Corannulene- & -16.05 & -10.37 & -37.41 & 40.63 & -22.40 \\
$\mathrm{H}_{2} \mathrm{O}^{20}$ & -21.61 & -6.07 & -23.03 & 32.85 & -17.87 \\
$\mathrm{ACE}-\mathrm{H}_{2} \mathrm{O}$ & -36.94 & -15.36 & -38.37 & 54.77 & -35.86 \\
Ace- $\left(\mathrm{H}_{2} \mathrm{O}\right)_{2}$ & -44.43 & -18.66 & -54.31 & 77.15 & -40.25 \\
Ace- $\left(\mathrm{H}_{2} \mathrm{O}\right)_{3}$ & -47.11 & -21.00 & -60.38 & 84.98 & -43.51 \\
Ace- $\left(\mathrm{H}_{2} \mathrm{O}\right)_{4}$ & - & & &
\end{tabular}

The polycyclic aromatic hydrocarbon acenaphthene has been successfully complexed with up to four water molecules, 
and the experimental structures of the water binding motifs on the PAH surface have been uncovered for Ace- $\left(\mathrm{H}_{2} \mathrm{O}\right)_{\mathrm{n}=1-3}$. We have seen that the water-water interactions on this mostly planar surface dominate, with water retaining most of the pure water cluster characteristics. The water clusters that bind to Ace remain intact due to the fact that they are largely bound by non-directional dispersion forces. This has allowed the first high resolution observations of a distorted cyclic water trimer complexed with an organic molecule. When compared to their pure water counterparts, the distortions incurred in the water moieties have been rationalized by a net cooperativity model that can be used for predicting the effects of water-substrate interactions in molecular aggregates of increasing size. As the size of the water cluster bound to the PAH increases, the dissociation energy increases, making it evident that they would be more likely to survive in astronomical environments and serve as a template for ice grain growth. The results presented here can be used to improve the accuracy of modeling reactions that may take place with solvated PAHs or on ice grain surfaces.

\section{ASSOCIATED CONTENT}

Supporting Information.

Rotational spectra, ab initio calculations, structural determination, hydrogen bond net cooperativity analysis, symmetry adapted perturbation theory calculations, and other experimental details

This material is available free of charge via the Internet at http://pubs.acs.org.

\section{AUTHOR INFORMATION}

\section{Corresponding Author}

* amanda.steber@mpsd.mpg.de

* berhane.temelso@ furman.edu

* $\underline{\text { melanie.schnell@mpsd.mpg.de }}$

\section{ACKNOWLEDGMENT}

This work was performed as part of our activities within the framework of the ERC Starting grant 'Astrorot', grant agreement number 638027. A.L.S. and A.M.R. were supported by the excellence cluster 'The Hamburg Centre for Ultrafast Imaging - Structure, Dynamics and Control of Matter at the Atomic Scale' of the Deutsche Forschungsgemeinschaft via a Louise Johnson Fellowship and a Mildred-Dresselhaus award, respectively. B. T. and G. C. S. acknowledge support by NSF grants CHE-1508556 and CHE-1721511, and by NSF grant CHE-1229354 and CHE1662030 as part of the MERCURY high-performance computer consortium. Z. K. acknowledges financial support from a grant from the Polish National Science Centre, decision number DEC/2011/02/A/ST2/00298.

\section{REFERENCES}

(1) Tielens, A. G. G. M. Annu. Rev. Astron. Astrophys. 2008, 46 (1), 289-337.

(2) Micelotta, E. R.; Jones, A. P.; Tielens, A. G. G. M. Astron. Astrophys. 2010, 510, A36.

(3) Boersma, C.; C. W. Bauschlicher, J.; Ricca, A.; Mattioda, A. L.; Cami, J.; Peeters, E.; Armas, F. S. de; Saborido, G. P.; Hudgins, D. M.; Allamandola, L. J. Astrophys. J. Suppl. Ser. 2014, 211 (1), 8.
(4) Joblin, C.; Mulas, G. EAS Publ. Ser. 2009, 35, 133-152.

(5) Allamandola, L. J.; Tielens, A. G. G. M.; Barker, J. R. Astrophys. J. Suppl. Ser. 1989, 71, 733-775.

(6) Cami, J.; Bernard-Salas, J.; Peeters, E.; Malek, S. E. Science 2010, 329 (5996), 1180-1182

(7) Bierbaum, V. M.; Le Page, V.; Snow, T. P. EAS Publ. Ser. 2011, 46, 427-440.

(8) Berné, O.; Tielens, A. G. G. M. Proc. Natl. Acad. Sci. 2012, 109 (2), 401-406.

(9) Shields, R. M.; Temelso, B.; Archer, K. A.; Morrell, T. E.; Shields, G. C. J. Phys. Chem. A 2010, 114 (43), 1172511737.

(10) Temelso, B.; Archer, K. A.; Shields, G. C. J. Phys. Chem. A 2011, 115 (43), 12034-12046.

(11) Dyke, T. R.; Muenter, J. S. J. Chem. Phys. 1974, 60 (7), 2929-2930.

(12) Pérez, C.; Muckle, M. T.; Zaleski, D. P.; Seifert, N. A.; Temelso, B.; Shields, G. C.; Kisiel, Z.; Pate, B. H. Science 2012, 336 (6083), 897-901.

(13) Pérez, C.; Lobsiger, S.; Seifert, N. A.; Zaleski, D. P.; Temelso, B.; Shields, G. C.; Kisiel, Z.; Pate, B. H. Chem. Phys. Lett. 2013, 571, 1-15.

(14) Pérez, C.; Zaleski, D. P.; Seifert, N. A.; Temelso, B.; Shields, G. C.; Kisiel, Z.; Pate, B. H. Angew. Chem. Int. Ed. 2014, 53 (52), 14368-14372.

(15) Keutsch, F. N.; Saykally, R. J. Proc. Natl. Acad. Sci. 2001, 98 (19), 10533-10540.

(16) Keutsch, F. N.; Cruzan, J. D.; Saykally, R. J. Chem. Rev. 2003, 103 (7), 2533-2578.

(17) Pugliano, N.; Saykally, R. J. Science 1992, 257 (5078), 1937-1940.

(18) Suzuki, S.; Green, P. G.; Bumgarner, R. E.; Dasgupta, S.; Goddard, W. A.; Blake, G. A. Science 1992, 257 (5072), 942-945.

(19) Gutowsky, H. S.; Emilsson, T.; Arunan, E. J. Chem. Phys. 1993, 99 (7), 4883-4893.

(20) Pérez, C.; Steber, A. L.; Rijs, A. M.; Temelso, B.; Shields, G. C.; Lopez, J. C.; Kisiel, Z.; Schnell, M. Phys. Chem. Chem. Phys. 2017, 19 (22), 14214-14223.

(21) Brown, G. G.; Dian, B. C.; Douglass, K. O.; Geyer, S. M.; Pate, B. H. J. Mol. Spectrosc. 2006, 238 (2), 200-212.

(22) Schnell, M. Z. Für Phys. Chem. 2012, 227 (1), 1-22.

(23) Kraitchman, J. Am. J. Phys. 1953, 21 (1), 17-24.

(24) Kisiel, Z. J. Mol. Spectrosc. 2003, 218 (1), 58-67.

(25) Watson, J. K. G.; Roytburg, A.; Ulrich, W. J. Mol. Spectrosc. 1999, 196 (1), 102-119.

(26) Thorwirth, S.; Theulé, P.; Gottlieb, C. A.; McCarthy, M. C.; Thaddeus, P. Astrophys. J. 2007, 662, 1309.

(27) Evangelisti, L.; Caminati, W. Phys. Chem. Chem. Phys. 2010, 12 (43), 14433-14441.

(28) Richardson, J. O.; Pérez, C.; Lobsiger, S.; Reid, A. A.; Temelso, B.; Shields, G. C.; Kisiel, Z.; Wales, D. J.; Pate, B. H.; Althorpe, S. C. Science 2016, 351 (6279), 1310-1313.

(29) Arunan, E.; Emilsson, T.; Gutowsky, H. S. J. Am. Chem. Soc. 1994, 116 (18), 8418-8419.

(30) Pérez, C.; Neill, J. L.; Muckle, M. T.; Zaleski, D. P.; Peña, I.; Lopez, J. C.; Alonso, J. L.; Pate, B. H. Angew. Chem. Int. Ed. 2015, 54 (3), 979-982.

(31) Ohno, K.; Okimura, M.; Akai, N.; Katsumoto, Y. Phys. Chem. Chem. Phys. 2005, 7 (16), 3005-3014.

(32) Tainter, C. J.; Skinner, J. L. J. Chem. Phys. 2012, 137 (10), 104304.

(33) Tabor, D. P.; Kusaka, R.; Walsh, P. S.; Zwier, T. S.; Sibert, E. L. J. Phys. Chem. A 2015, 119 (38), 9917-9930.

(34) Tabor, D. P.; Kusaka, R.; Walsh, P. S.; Sibert, E. L.; Zwier, T. S. J. Phys. Chem. Lett. 2015, 6 (10), 1989-1995.

(35) Jeziorski, B.; Moszynski, R.; Szalewicz, K. Chem. Rev. 1994, 94 (7), 1887-1930. 
Authors are required to submit a graphic entry for the Table of Contents (TOC) that, in conjunction with the manuscript title, should give the reader a representative idea of one of the following: A key structure, reaction, equation, concept, or theorem, etc., that is discussed in the manuscript. Consult the journal's Instructions for Authors for TOC graphic specifications.

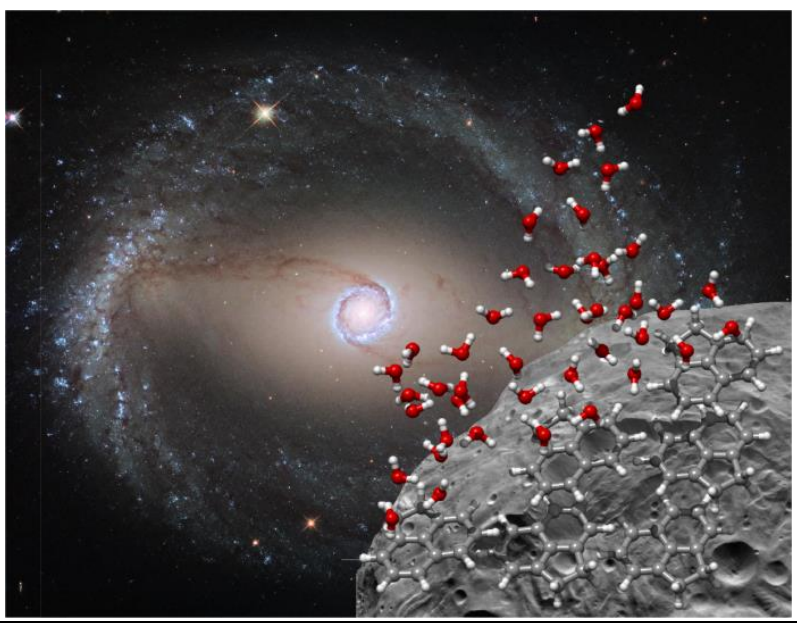

